\title{
An Effective and Easy-to-Implement Boundary Condition for Molecular Dynamics Simulations
}

\author{
Cheng Yuan ${ }^{1}$, Jerry Zhijian Yang ${ }^{1,2, *}$ and Xiantao $\mathrm{Li}^{3}$ \\ 1 School of Mathematics and Statistics, Wuhan University, Wuhan, Hubei 430072, \\ P.R. China. \\ ${ }^{2}$ Hubei Key Laboratory in Computational Mathematics (Wuhan University), Wuhan, \\ Hubei 430072, P.R. China. \\ ${ }^{3}$ Department of Mathematics, The Pennsylvania State University, University Park, \\ PA 16802, USA.
}

Received 17 November 2018; Accepted (in revised version) 16 January 2019

\begin{abstract}
This paper presents an absorbing boundary condition for molecular dynamics simulations of materials defects. The purpose of the boundary condition is to eliminates spurious reflections of phonons at the boundary and minimize the finite size effect. In contrast to other existing methods, our emphasis is placed on the ease of implementation. In particular, we propose a method for which the implementation can be done within existing molecular dynamics code, and it is insensitive to lattice structure, the geometry and space dimension of the computational domain. To demonstrate the effectiveness, the results from two test problems are presented.
\end{abstract}

AMS subject classifications: 70-08

Key words: Molecular dynamics, boundary conditions, dislocation, crack, Krylov space.

\section{Introduction}

Molecular dynamics (MD) models have emerged in the last few decades as a powerful methodology in studying material properties. In MD, the interactions of the atoms are explicitly taken into account [1]. As a result, assumptions on the strain-stress relation, interface kinetics, geometry of crystal defects etc, are not needed. Expressed as secondorder ordinary differential equations (ODEs), the numerical implementation has been standardized, e.g. see [1]. Recent development of more accurate interatomic potentials [2-4] has made MD even more promising. In particular, MD models that are based on fundamental considerations (quantum mechanics) are also available [5].

*Corresponding author. Email addresses: zjyang.math@whu.edu.cn (J. Z. Yang), yuancheng@whu.edu.cn (C. Yuan), xli@math.psu.edu (X. Li) 
Meanwhile, a fundamental limitation, which will likely remain for many years to come, is the scale of the material systems that it can describe. This difficulty stems from the intrinsic spatial scale of MD, which starts at the Angstrom scale $\left(10^{-10} \mathrm{~m}\right)$. As a result, one is often restricted to small systems (e.g., thousands to a few million atoms). In addition, periodic boundary conditions (PBC) have been implemented almost exclusively. The implication of PBC is an infinite system with periodic images, which creates an artificial interactions among the images. Furthermore, applying non-uniform boundary condition under this setting is not possible. More importantly, there are many mechanical systems that can not be embedded into a larger periodic sample, e.g, an open crack.

Fortunately, non-periodic BCs that correctly describe the influences of the surrounding medium has been recently developed by numerous groups [6-10]. The mathematical derivation starts with an infinite system, from which the computational domain is selected. The degrees of freedom associated with the atoms in the surrounding region are eliminated by using Fourier/Laplace transform. The result is that the atoms outside the boundary can be written as an integral that involves the time history of displacement of the atoms inside the computational domain. In principle this gives the correct boundary condition. For planar boundaries, this derivation is particularly simple, and it can be implemented directly [10-12]. In this case, the BCs can also be approximated by minimizing the reflection coefficients $[6,7,9,13-15]$. Another important application of these BCs can be found in multiscale models that involve MD and continuum models [16-24] to reduce the computational cost. Typical observations are that as defects migrate, lattice waves are generated continuously, and as they arrive at the artificial boundary, reflections may occur. In many cases, the reflected waves interfere with the dynamics of the defects e.g., [25], which is undesirable. This observation is similar to artificial reflections in the simulations of wave propagation problems, where absorbing BCs play a vital role in minimizing the boundary reflection [26-28].

Despite the success of the existing methods, there are several remaining difficulties. First, the implementation of most existing methods requires significant effort to pre-compute the parameters. For example, such preparations include the force constant matrices, crystal orientations, and the geometry of the computational domain. These steps may not be familiar to general MD practitioners. Secondly, most of the calculations are limited to planar boundaries, and the proper extension to corners is highly nontrivial $[9,11]$. Thirdly, when the BC is expressed as a memory integral, one needs to store the previous values of the displacement of the atoms near the boundary, and the integrals have to be evaluated at every step in time. This significantly increases the computational cost. Finally, the implementation of the BCs in existing code is not straight-forward. To our knowledge, none of the above mentioned methods has been included in existing MD code.

This paper presents a simple approach to implement an approximation of the absorbing BCs. We first express the exact $\mathrm{BC}$ as a convolutional integral where the kernel function is written as a matrix function. Motivated by the success of the Krylov subspace approximation of matrix functions [29-32], we introduce a hierarchical approximation. 
We then show that this approximation is equivalent to extending the system by adding a few auxiliary variables. This will effectively eliminate the history dependence and makes the implementation much more efficient. What makes this method particularly appealing is that the implementation can be easily incorporated into an existing MD code. In particular, the subspace only requires force calculations, which are usual components in MD. The method is insensitive to the lattice structure, the geometry and the dimensionality of the computational domain. Finally, the extended system inherits the equation forms of the Newton's equations of motion. Consequently, the Verlet type of integrators can be used.

The rest of the paper is organized as follows. We first show a derivation of the BC using a matrix-vector notation. Then in Section 2, we present an approximation of the memory function using Krylov subspaces. The implementation of this algorithm using force calculation subroutines are then discussed in Section 3. Finally, we present results from several numerical tests.

\section{A Mathematical formulation of the boundary condition}

We have in mind a system of $N$ atoms, and we divide the system into two subdomains. The first sub-domain, denoted by $\Omega_{I}$, contains the defects of interest, and the surrounding region, denoted by $\Omega_{I I}$, can be regarded as an elastic medium surrounding the defects. The partition of the domain is illustrated in Fig. 1. Meanwhile, we denote the displacement of all the atoms by $u$ with reference position $X$, and $u$ is determined by Newton's equations of motion,

$$
m_{i} \ddot{u}_{i}=-\frac{\partial U}{\partial u_{i}} .
$$

For simplicity of the notations, the mass $m_{i}$ will be set to unity. Here $U\left(\boldsymbol{u}_{1}, \boldsymbol{u}_{2}, \cdots\right)$ is the potential energy that describes the interaction among the atoms. However, the method-

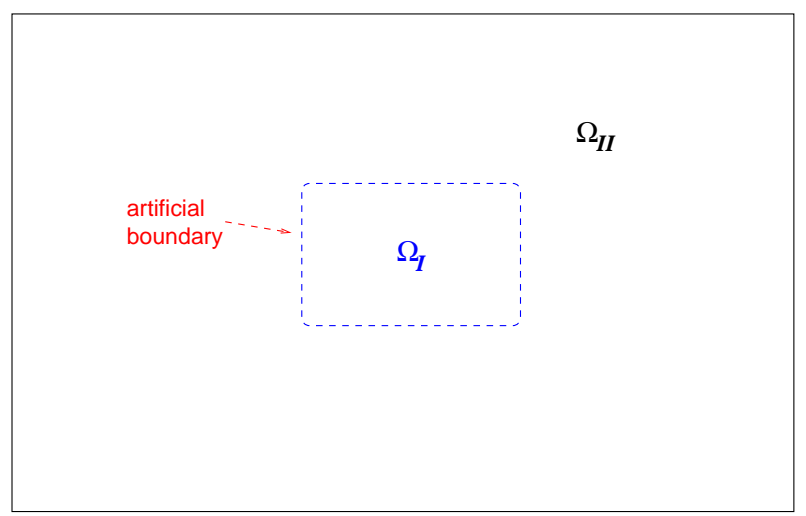

Figure 1: A schematic of the partition of the domain. The system contains $N$ total atoms and $n$ atoms are selected to create the computational domain $\Omega_{I}, n \ll N$. 
ology presented in this paper will not depend on the specific form of $U$. Most empirical potentials for solids are short-ranged: The interaction is zero when the atomic distance is beyond a cut-off radius $r_{\text {cut }}$. This will be assumed in the present approach.

\subsection{The exact boundary condition}

In accordance to the partition of the domain, we divide the atomic displacement into $\left(\boldsymbol{u}_{I}, \boldsymbol{u}_{I I}\right)$, representing respectively the displacement for the atoms inside and outside the actual computational domain $\Omega_{I I}$. Further, we divide the system of equations (2.1) into two groups, and they are written in the following form,

$$
\begin{aligned}
& \ddot{\boldsymbol{u}}_{I}=-\frac{\partial U}{\partial \boldsymbol{u}_{I}}\left(\boldsymbol{u}_{I}, \boldsymbol{u}_{I I}\right), \\
& \ddot{\boldsymbol{u}}_{I I}=-K_{I I, I} \boldsymbol{u}_{I}-K_{I I, I I} \boldsymbol{u}_{I I} .
\end{aligned}
$$

We will assume that initially

$$
\dot{\boldsymbol{u}}_{I I}(0)=\boldsymbol{u}_{I I}(0)=0 .
$$

This means that initially the surrounding region is in a mechanical equilibrium and it is a typical assumption for deriving an absorbing BC.

In addition, we have linearized the interactions that involve the atoms in the surrounding region $\Omega_{I I}$. The coefficients $K_{I, I I} \in \mathbb{R}^{n \times(N-n)}, K_{I I, I}\left(=K_{I, I I}^{T}\right)$ and $K_{I I, I I} \in$ $\mathbb{R}^{(N-n) \times(N-n)}$ contain the force constant matrices. More specifically, we have

$$
\begin{aligned}
& K_{I, I I}=\frac{\partial^{2} U}{\partial u_{I} \partial u_{I I}}, \\
& K_{I I, I I}=\frac{\partial^{2} U}{\partial u_{I I}^{2}} .
\end{aligned}
$$

The linearization can be justified on the ground that away from defects, the atomic displacement is smooth.

Eqs. (2.2), (2.3) and (2.4) have been the starting point to derive explicit BCs for MD models $[9-12,33]$. We now briefly show a derivation in a matrix-vector form. By solving the second equation in (2.2), we find that

$$
\boldsymbol{u}_{I I}(t)=\int_{0}^{t} K_{I I, I I}^{-\frac{1}{2}} \sin \left[\sqrt{K_{I I, I I}}(t-s)\right] K_{I I, I} \boldsymbol{u}_{I}(s) d s .
$$

The trigonometric matrix function is defined as

$$
\sin A=A-\frac{1}{3 !} A^{3}+\frac{1}{5 !} A^{5} \cdots
$$

Namely, it is defined based on the Taylor series. One can easily verify from this expansion that the kernel function in (2.5) only involves the integer powers of the matrix $K_{I I, I I}$. 
This BC expresses the displacement of the atoms in $\Omega_{I I}$ in terms of the history of the displacement of the atoms in the interior $\Omega_{I}$. At least for the model (2.2), this BC is exact. The main difficulty is due to the memory function, which has been expressed as a matrix function. Due to the large dimension of $K_{I I, I I}$, direct computation is impractical. Next we present a systematic approach to approximate (2.5).

\subsection{Krylov subspace approximation of the boundary condition}

The BC (2.5) involves a matrix function. A well established method for approximating matrix functions is the Krylov subspace approximation [29-32]. For the present problem, due to the short range interaction, the matrix $K_{I I, I}$ is sparse: Only the entries corresponding to the atoms at the artificial boundary are non-zero. This makes the Krylov subspace approximation particularly efficient. More specifically, we let $V$ be the columns of $K_{I I, I}$ that correspond to the those inside atoms whose distance to the boundary is less than $r_{\text {cut }}$. This set of atoms are specifically defined as follows,

$$
B^{-}=\left\{X_{i} \in \Omega_{I}, \operatorname{dist}\left(X_{i}, \Omega_{I I}\right) \leq r_{\text {cut }} \cdot\right\} .
$$

With these preparations, we let $A=K_{I I, I I}$, and define the block Krylov subspace:

$$
\mathcal{K}_{\ell}(A ; V)=\operatorname{span}\left\{V, A V, \cdots, A^{\ell} V\right\} .
$$

The implementation of the Krylov subspace requires a set of vectors that form the basis of the subspace. This is typically done by using the block Lanczos method [31,32]. Let $m$ be the rank of $V$. The block Lanczos algorithm is as follows.

Algorithm 1. (Block Lanczos). Set $V_{0}=0, Z_{0}=V$ and $p_{0}=m$. For $j=1,2, \cdots, \ell$, repeat:

Rank revealing QR factorization [34] of the matrix $Z_{j-1}: Z_{j-1}=Q_{j} R_{j-1}$.

- Let $p_{j}=\operatorname{rank}\left(Z_{j-1}\right), V_{j}$ be the first $p_{j}$ columns of $Q_{j}$, and $B_{j-1}$ be the first $p_{j}$ rows of $R_{j-1}$;

- $Z_{j} \longleftarrow A V_{j}-V_{j-1} B_{j-1}^{T}$;

- $A_{j} \longleftarrow V_{j}^{T} Z_{j}$

- $Z_{j} \longleftarrow Z_{j}-V_{j} A_{j}$.

Let us write the basis vectors in the form of,

$$
Q=\left[Q_{0} Q_{1} \cdots Q_{\ell}\right] \text {. }
$$

Let $M$ be the number of column in $Q$. It is the dimension of the subspace. Clearly, $M \leq(\ell+1) m$. In particular, $Q_{0}$ can be obtained from a $\mathrm{QR}$ decomposition of $V$

$$
V=Q_{0} B_{0},
$$


in which $B_{0}$ is a upper triangular matrix. Our experiences suggest that $2 \leq \ell \leq 6$ is usually adequate.

Of particular importance to the matrix function approximation is the fact that

$$
A Q \approx Q T,
$$

where $T \in \mathbb{R}^{M \times M}$ is a block tri-diagonal matrix. As a result of this approximation, we can approximate the product of a matrix function $f(A)$ and the vector $Q$ as

$$
f(A) Q \approx Q f(T) .
$$

Since $T$ is a block tri-diagonal matrix with much smaller dimension, the right hand side can be efficiently computed.

In particular, the $\mathrm{BC}(2.5)$ can be approximated by

$$
\boldsymbol{u}_{I I}(t) \approx \int_{0}^{t} Q T^{-\frac{1}{2}} \sin \left[T^{\frac{1}{2}}(t-s)\right] E_{1}^{T} \boldsymbol{u}_{I}(s) d s .
$$

Here $E_{1}$ has a similar block structure as $Q: E_{1}=\left[B_{0}^{T} 00 \cdots 0\right]$.

Despite the availability of the exact solution (2.5) and the reduction to the simpler form (2.12), there are several remaining practical issues:

1. The force constant matrices that form the matrix $A$ need to be computed a priori. For artificial boundary with general geometry, this procedure can be quite cumbersome.

2. The dimension of $A$, which is determined by the size of $\Omega_{I I}$, is too large.

3. In order to implement (2.12), the history of $\boldsymbol{u}_{I}$ has to be stored. The integral has to be evaluated at every step. This leads to significant computational overhead.

Due to these issues, implementing (2.12) within an existing MD code would still require significant revision and additions. In the next section, we show a much simplified implementation method.

\section{An alternative implementation}

\subsection{The evaluation of the force constant matrices in the Krylov subspace}

Here we propose an algorithm that can be easily implemented in existing MD code. First, we notice that the force constant matrices (2.4) can be computed using a finite difference approximation using existing force calculation routines, which is usually available in MD packages. In particular, let $f=-\nabla U$, then we have

$$
K_{i \alpha, j \beta} \approx-\frac{f_{j \beta}\left(X+\epsilon e_{i \alpha}\right)-f_{j \beta}\left(X-\epsilon e_{i \alpha}\right)}{2 \epsilon} .
$$


Here $j \beta$ labels the $\beta$-th component of the force on the $j$-th atom, and $e_{i \alpha}$ is the unit vector whose $i \alpha$-th component is one and all other components are zero.

Using Eq. (3.1), one can compute the initial vector $V$. The Krylov subspace also involves matrix multiplications, e.g., $A Y$, with $Y$ being any matrix. This can be approximated in the same way. Namely,

$$
A \boldsymbol{y}_{i} \approx-\frac{\mathbf{f}\left(X+\epsilon \boldsymbol{y}_{i}\right)-\mathbf{f}\left(X-\epsilon \boldsymbol{y}_{i}\right)}{2 \epsilon}
$$

where $y_{i}$ is the $i$-th column of the matrix $Y$.

Due to the short range interaction, the matrix entry $K_{i \alpha, j \beta}$ is only non-zero for atoms near the artificial boundary. Therefore, the perturbation of the $i$ th atom only needs to be introduced for atoms that are inside, but close to the boundary, i.e., that atoms in $B^{-}$.

Thanks again to the short range of the interatomic potential, the matrix $A$ will have band structure with bandwidth consistent with the cut-off radius. As a result, the vectors $A^{\ell} V$ are only confined to a few layers outside the boundary. These atoms can be found in the set

$$
B^{+}=\left\{X_{i} \in \Omega_{I I}, \operatorname{dist}\left(X_{i}, \Omega_{I}\right) \leq \ell r_{\text {cut }}\right\} .
$$

In our algorithm we only evaluate the forces on those atoms, which significantly reduces the computational cost associated with the matrix vector multiplications.

We further comment that these calculations are performed prior to the simulation. They only need to be done once.

\subsection{An extended system with no memory}

We now address the issue with the memory dependence. An important observation is that when $\boldsymbol{u}_{I I}$ is approximated in the subspace (2.8),

$$
\boldsymbol{u}_{I I}=Q z(t),
$$

the second equation in (2.2) is reduced to

$$
\ddot{z}=-E_{1}^{T} \boldsymbol{u}_{I}-T \boldsymbol{z}(t) .
$$

Here $z \in \mathbb{R}^{M}$ is the coefficients of $\boldsymbol{u}_{I I}$ in the subspace.

Now we can simply solve the equation for $z(t)$ and use (3.4) to reconstruct the position of the atoms outside the boundary. We also notice that: (1.) The dimension of $z$ equals the dimension of the subspace, which is typically very small compared to the dimension of the entire problem; (2.) Since the matrix $E_{1}$ has many zero entries, the interaction term can be easily computed. More importantly, one can easily show that the models (3.4) and (3.5) are equivalent to the approximate BC (2.12).

Next, to incorporate this observation to an existing MD code, we will replace the equation (3.5) by a MD-like nonlinear model. To be more specific, we start from the fully nonlinear model for the atoms outside,

$$
\ddot{u}_{I I}=f_{I I}\left(u_{I}, u_{I I}\right),
$$


whose linearization corresponds to the second equation in (2.2). We now bypass the linearization step, which led to the second equation in (2.2), by projecting this nonlinear equation to the Krylov subspace. Namely, we seek $\boldsymbol{u}_{I I}=Q z$, such that

$$
\left(\ddot{\boldsymbol{u}}_{I I}, \boldsymbol{q}\right)=\left(f_{I I}, \boldsymbol{q}\right)
$$

for any $q$ in the subspace, especially the columns of $Q$.

A direct substitution yields

$$
\ddot{z}=Q^{T} f_{I I}\left(\boldsymbol{u}_{I}, Q z\right) .
$$

In the case when $f_{I I}$ is linearized (cf. the second equation in (2.2)), we would have arrived at Eq. (3.5), i.e., they are equivalent in the linearized case. But it is clear that (3.8) is much easier to implement in an existing MD code. More importantly, since the basis vectors in $Q$ are localized and the support is confined to the boundary (the set $B^{+}$), only the components of $f_{I I}$ that correspond to the forces on the atoms near the boundary need to be computed (3.8). Another advantage is that the extended system (3.8) is again a system of second-order differential equations with no memory.

Finally, the algorithm can be implemented as follows.

Algorithm 2. (Implementation).

1. Define the atoms outside $(I I)$ and inside $(I)$ the boundary.

2. Use finite difference to generate the matrix $V$.

3. Use the Lanczos algorithm to generate the orthogonal basis $Q$.

4. Time integration: set $z=0, \eta(=\dot{z})=0$. For $n=0,1, \cdots$,

(a) $\boldsymbol{v}_{I} \rightarrow \boldsymbol{v}_{I}+f_{I} \Delta t / 2 ; \quad \boldsymbol{\eta} \rightarrow \boldsymbol{\eta}+Q^{T} f_{I I} \Delta t / 2 ;$

(b) $\boldsymbol{u}_{I} \rightarrow \boldsymbol{u}_{I}+\boldsymbol{v}_{I} \Delta t ; \quad z \rightarrow z+\eta \Delta t$;

(c) $\boldsymbol{u}_{I I}=Q z$;

(d) Compute the forces $f$ (and separate $f_{I}$ and $f_{I I}$ );

(e) $\boldsymbol{v}_{I} \rightarrow \boldsymbol{v}_{I}+f_{I} \Delta t / 2 ; \quad \boldsymbol{\eta} \rightarrow \boldsymbol{\eta}+Q^{T} f_{I I} \Delta t / 2$.

Meanwhile, since our primary interest is in the variable $\boldsymbol{u}_{I}$ and the information outside $\Omega_{I}$ is only designed as absorbing boundary conditions, we can practically introduce a damping term in the equation of $z$ to avoid a delayed spurious reflection at boundary due to the inaccuracy of Krylov subspace approximation and the outmost boundary condition of $\Omega_{I I}$. We introduce a damping term in the equation of $z$, namely,

$$
\ddot{z}=Q^{T} f_{I I}\left(\boldsymbol{u}_{I}, Q \boldsymbol{z}\right)-\gamma \boldsymbol{\eta} .
$$

We change step 4(a) and 4(e) to

$$
\boldsymbol{v}_{I} \rightarrow \boldsymbol{v}_{I}+f_{I} \Delta t / 2 ; \quad \eta \rightarrow \eta+Q^{T} f_{I I} \Delta t / 2-\gamma \eta \Delta t / 2 .
$$




\section{Numerical examples}

In this section we will give two numerical examples to test the new MD boundary conditions. The first example is an implementation of the $\mathrm{BC}$ s for lattice waves generated by an edge dislocation, and the second is for the waves around a crack. In both cases we could see that the reflected wave at the boundary can be well absorbed by our new boundary condition.

\subsection{An example for a dislocation}

In this example we consider the dislocation dipole problem in a face-centered-cubic (fcc) crystal of aluminum. We use the embedded atom potential in [3] as the atomic potential $U$.

We study a rectangular system with the three axes along [110],[001],[110] directions respectively, which contains $100 \times 60$ primitive cells on the plane with periodic boundary condition in the third direction to model a plain strain condition. $\Omega_{I}$ contains 7872 atoms, and the remain few layers of atoms in the surrounding area are identified as $\Omega_{I I}$. To initialize the dislocation, we use the analytical solution of the linear elasticity equation for an edge dislocation [35] and prescribe the displacement as follows,

$$
\left\{\begin{array}{l}
u_{x}(x, y)=b / 2 \pi\left[\theta(x, y)+\frac{x y}{2(1-v)\left(x^{2}+y^{2}\right)}\right] \\
u_{y}(x, y)=-b / 2 \pi\left[\frac{1-2 v}{4(1-v)} \ln \left(x^{2}+y^{2}\right)+\frac{x^{2}-y^{2}}{4(1-v)\left(x^{2}+y^{2}\right)}\right] \\
u_{z}(x, y)=0,
\end{array}\right.
$$

where the dislocation line direction is $\boldsymbol{e}_{z}$ and the Burgers vector is $\boldsymbol{b}=\boldsymbol{b} \boldsymbol{e}_{x}$. Notice that the $(x, y)$ here refers to the position relative to the dislocation core and the $\theta(x, y)$ is the angle between $\boldsymbol{e}_{x}$ and $(x, y, 0)$.

For comparison purposes, we also implemented a fixed boundary condition, where we simply hold that atoms at the boundary according to (4.1). As shown in Fig. 2, the new boundary condition with the Krylov subspace may indeed eliminate the reflected wave at the artificial boundary, and as we have mentioned, the complexity of updating has been largely decreased since we project a much higher dimensional vector $\boldsymbol{u}_{I I}$ to the Krylov subspace. Here, we choose $\ell=2$. The dimension of the Krylov subspace is 2676 .

\subsection{An example for an elliptic crack}

For the second test, we keep all the numerical setup mentioned in the previous case, except we would displace the atoms according to the displacement given by [36]: 


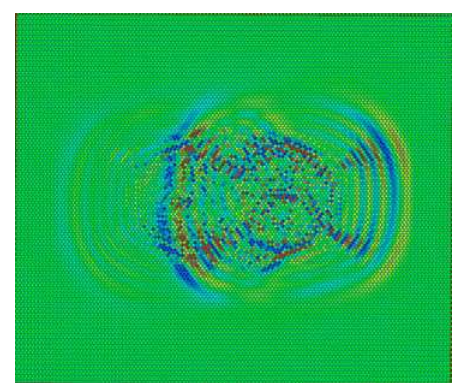

(fixed BC, $t=0.795 \mathrm{ps}$ )

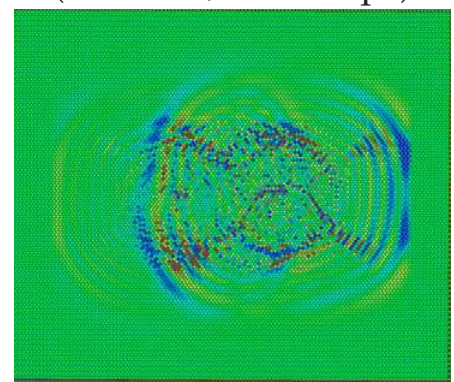

(fixed BC, $t=1.06 \mathrm{ps}$ )

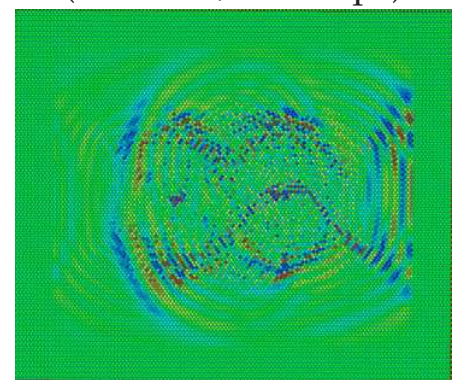

(fixed BC, $t=1.325 \mathrm{ps}$ )

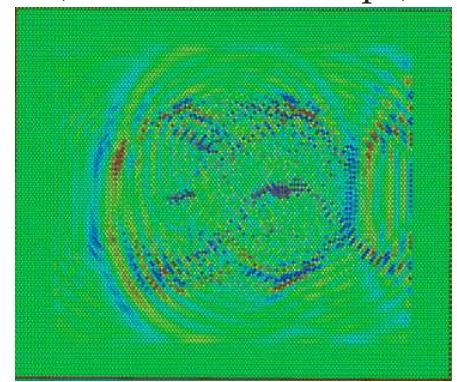

(fixed BC, $t=1.59 \mathrm{ps}$ )

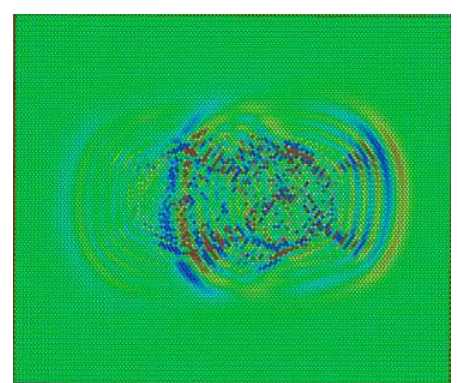

(new BC, $t=0.795 \mathrm{ps}$ )

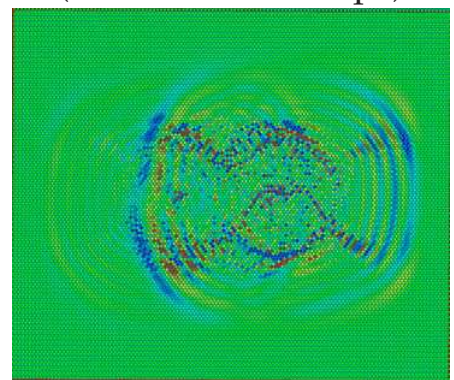

(new $\mathrm{BC}, t=1.06 \mathrm{ps}$ )

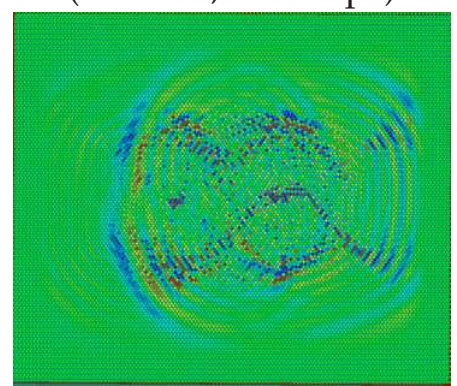

(new BC, $t=1.325 \mathrm{ps}$ )

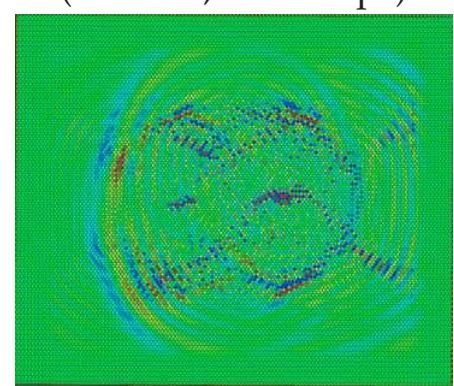

(new $\mathrm{BC}, t=1.59 \mathrm{ps}$ )

Figure 2: Fixed boundary condition and new boundary condition with Krylov subspace for dislocation dipole. We could see the dislocation waves are reflected at the boundary if we use fixed boundary condition, while the wave could be well absorbed with our new boundary condition. 


$$
\left\{\begin{aligned}
u_{x}(x, y)= & K_{I}\left(\frac{2 r}{\pi}\right)^{1 / 2} \operatorname{Re}\left\{\frac { 1 } { s _ { 1 } - s _ { 2 } } \left[s_{1} p_{2}\left(\cos \theta+s_{2} \sin \theta\right)^{1 / 2}\right.\right. \\
& \left.\left.-s_{2} p_{1}\left(\cos \theta+s_{1} \sin \theta\right)^{1 / 2}\right]\right\}, \\
u_{y}(x, y)= & K_{I}\left(\frac{2 r}{\pi}\right)^{1 / 2} \operatorname{Re}\left\{\frac { 1 } { s _ { 1 } - s _ { 2 } } \left[s_{1} q_{2}\left(\cos \theta+s_{2} \sin \theta\right)^{1 / 2}\right.\right. \\
& \left.\left.-s_{2} q_{1}\left(\cos \theta+s_{1} \sin \theta\right)^{1 / 2}\right]\right\}, \\
u_{z}(x, y)= & 0,
\end{aligned}\right.
$$

where $r$ and $\theta$ are the polar coordinates and functions $p_{i}$ and $q_{i}$ are defined as

$$
\left\{\begin{array}{l}
p_{1}=a_{11} s_{1}^{2}+a_{12}-a_{16} s_{1} \\
p_{2}=a_{11} s_{2}^{2}+a_{12}-a_{16} s_{2} \\
q_{1}=\frac{a_{12} s_{1}^{2}+a_{22}-a_{26} s_{1}}{s_{1}} \\
q_{2}=\frac{a_{12} s_{2}^{2}+a_{22}-a_{26} s_{2}}{s_{2}}
\end{array}\right.
$$

Further, $s_{i}$ are two conjugate roots of a characteristic equation [36] and $a_{i j}$ are related to the elastic compliance constants of the material.

For the Krylov subspace, we choose $\ell=2$ again and the dimension of the Krylov space is still 2676. From Fig. 3, we observe that the crack propagates toward the boundary, and after the waves reached the boundary, they are reflected when the fixed BC is used. More reflections are observed from the top and bottom boundaries. On the other hand, with the new $\mathrm{BC}$, the waves coming from the crack tip have been well absorbed.

\section{Conclusions}

We have presented a new approach to formulate approximate boundary conditions for molecular dynamics simulations in solids. The particular emphasis has been placed on the effort of the implementation: All the steps have been designed so that they can be implemented within an MD code. For instance, the matrix multiplication in the Lanczos algorithm can be achieved by using the force calculation routines in MD. In addition, the boundary condition is reformulated into a second order ODE, which has the same form as the full MD model. Therefore, the time integration can be implemented using Verletlike algorithms. Compared to existing methods, this current formulation is much more accessible. An important effort would be to include this method to a MD software, such as LAMMPS [37]. This will be done in our future works, which also include the extension to finite temperature boundary conditions $[33,38]$. 

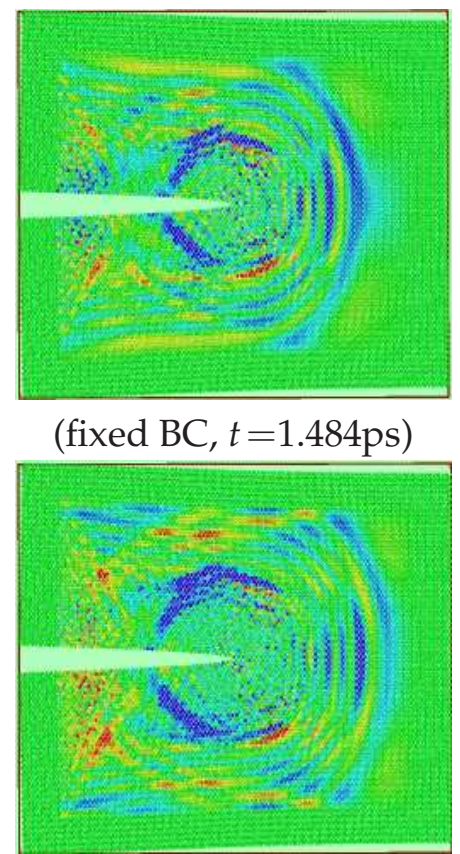

(fixed BC, $t=1.802 \mathrm{ps}$ )

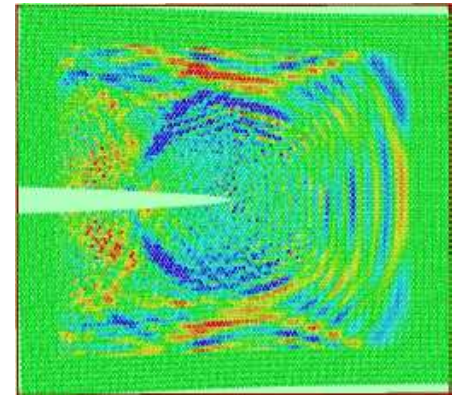

(fixed BC, $t=2.12 \mathrm{ps}$ )

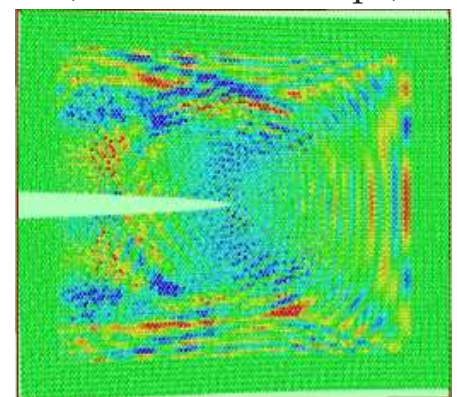

(fixed BC, $t=2.438 \mathrm{ps}$ )

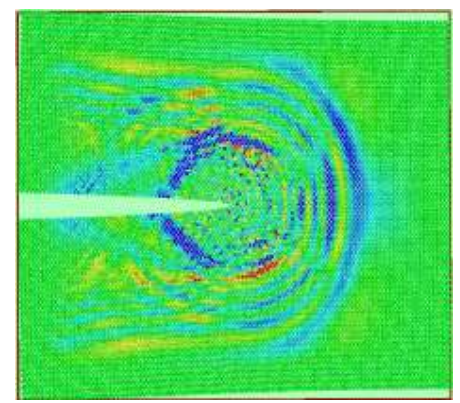

(new BC, $t=1.484 \mathrm{ps}$ )

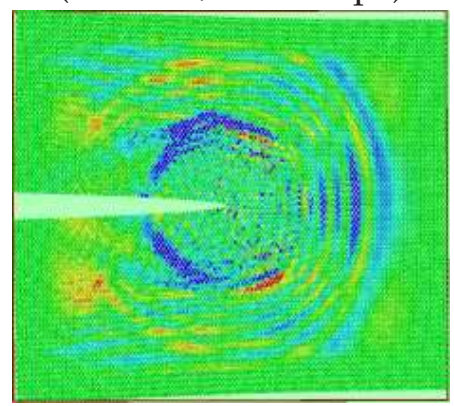

(new BC, $t=1.802 \mathrm{ps}$ )

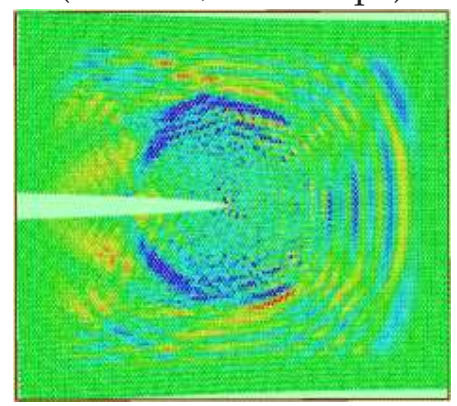

(new BC, $t=2.12 \mathrm{ps}$ )

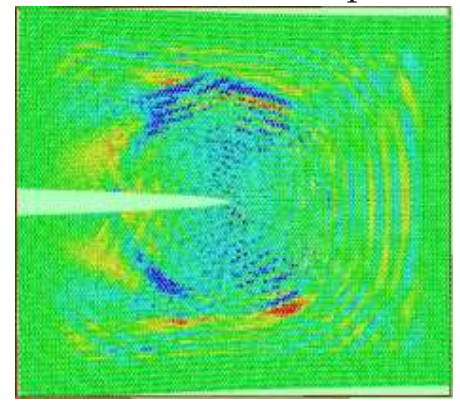

(new $\mathrm{BC}, t=2.438 \mathrm{ps}$ )

Figure 3: Numerical results for the crack propagation. Left: fixed BC; Right: the new BC. 


\section{References}

[1] D. Frenkel, B. Smit, Understanding molecular simulation: from algorithms to applications, 2nd Edition, Academic Press, 2002.

[2] H. Chamati, N. Papanicolaou, Y. Mishin, D. Papaconstantopoulos, Embedded-atom potential for fe and its application to self-diffusion on fe (100), Surface Science 600 (9) (2006) 17931803.

[3] F. Ercolessi, J. B. Adams, Interatomic potentials from first-principles calculations: the forcematching method, EPL (Europhysics Letters) 26 (8) (1994) 583.

[4] Y. Mishin, M. Mehl, D. Papaconstantopoulos, A. Voter, J. Kress, Structural stability and lattice defects in copper: Ab initio, tight-binding, and embedded-atom calculations, Physical Review B 63 (22) (2001) 224106.

[5] R. Car, M. Parrinello, Unified approach for molecular dynamics and density-functional theory, Physical review letters 55 (22) (1985) 2471.

[6] W. E, Z. Huang, A dynamic atomistic-continuum method for the simulation of crystalline material, Journal of Computational Physics 182 (2002) 234-261.

[7] W. E, Z. Huang, Matching conditions in atomistic-continuum modeling of material, Physical Review Letter 87 (2001) 135501.

[8] S. Li, X. Liu, A. Agrawal, A. C. To, Perfectly matched multiscale simulations for discrete lattice systems: Extension to multiple dimensions, Phys. Rev. B 74 (2006) 045418.

[9] X. Li, W. E, Variational boundary conditions for molecular dynamics simulations of solids at low temperature, Comm. Comp. Phys. 1 (2006) 136-176.

[10] G. J. Wagner, E. G. Karpov, W. K. Liu, Molecular dynamics boundary conditions for regular crystal lattice, Comp. Method Appl. Mech. Eng. 193 (2004) 1579-1601.

[11] E. Karpov, H. Yu, H. Park, W. K. Liu, Q. J. Wang, D. Qian, Multiscale boundary conditions in crystalline solids: Theory and application to nanoindentation, International Journal of Solids and Structures 43 (2006) 6359-6379.

[12] E. G. Karpov, G. J. Wagner, W. K. Liu, A Green's function approach to deriving non-reflecting boundary conditions in molecular dynamics simulations, Int. J. Numer. Meth. in Engng. 62 (9) (2005) 1250-1262.

[13] W. Cai, M. de Koning, V. V. Bulatov, S. Yip, Minimizing boundary reflections in coupleddomain simulations, Physical Review Letter 85 (2000) 3213.

[14] M. N. Guddati, S. Thirunavukkarasu, Phonon absorbing boundary conditions for molecular dynamics, J. Comp. Phys. 228 (2009) 8112-8134.

[15] S. Tang, A finite difference approach with velocity interfacial conditions for multiscale computations of crystalline solids, Journal of Computational Physics 227 (8) (2008) 4038-4062.

[16] J. Li, D. Liao, S. Yip, Coupling continuum to molecular-dynamics simulation: Reflecting particle method and the field estimator, Phys. Rev. E 57 (1998) 7259-7267. doi:10.1103/PhysRevE.57.7259.

[17] S. Tang, T. Y. Hou, W. K. Liu, A mathematical framework of the bridging scale method, Int. J. Numer. Meth. Engng. 65 (2006) 1688-1713.

[18] G. J. Wagner, W. K. Liu, Coupling of atomistic and continuum simulations using a bridging scale decomposition, Journal of Computational Physics 190 (2003) 249-274.

[19] J. Z. Yang, X. Li, Comparative study of boundary conditions for molecular dynamics simulations of solids at low temperature, Physical Review B 73 (22) (2006) 224111.

[20] J. T. Oden, S. Prudhomme, A. Romkes, P. Bauman, Multi-scale modeling of physical phenomena: Adaptive control of models, SIAM Journal on Scientific Computing 28(6) (2006) 
2359-2389.

[21] M. Parks, P. Bochev, R. Lehoucq, Connecting atomistic-to-continuum coupling and domain decomposition, Multiscale Model. Simul. 7 (1) (2008) 362-380.

[22] D. Rodney, Mixed atomistic/continuum methods: static and dynamic quasicontinuum methods, in: Proceedings of the NATO Conference: Thermodynamics, Microstructures and Plasticity, Finel, A., Maziere, D., and Veron, M. (eds.), Kluwer Academic Publishers,Netherlands, 2003, pp. 265-274.

[23] S. Xiao, T. Belytschko, A bridging domain method for coupling continua with molecular dynamics, Comput. Methods Appl. Mech. Engrg. 193 (2004) 1645-1669.

[24] L. Xiong, G. Tucker, D. L. McDowell, Y. Chen, Coarse-grained atomistic simulations of dislocations, J. Mech. Phys. Solids 59 (2011) 160-177.

[25] B. L. Holian, R. Ravelo, Fracture simulation using large-scale molecular dynamics, Physical Review B 51 (1995) 11275.

[26] B. Engquist, A. Majda, Absorbing boundary conditions for the numerical simulation of waves, Math. Comp. 31 (1977) 629-651.

[27] T. Hagstrom, Radiation boundary conditions for the numerical simulation of waves, Acta Numerica 8 (1999) 47-106.

[28] R. L. Higdon, Absorbing boundary conditions for difference approximations to the multidimensional wave equation, Math. Comp. 47 (1985) 437-459.

[29] M. Eiermann, O. G. Ernst, A restarted Krylov subspace method for the evaluation of matrix functions, SIAM Journal on Numerical Analysis 44 (6) (2006) 2481-2504. doi:10.1137/050633846. URL http://epubs.siam.org/doi/abs/10.1137/050633846

[30] L. Lopez, V. Simoncini, Analysis of projection methods for rational function approximation to the matrix exponential, SIAM Journal on Numerical Analysis 44 (2) (2006) 613-635. doi:10.1137/05062590.

URL http://epubs.siam.org/doi/abs/10.1137/05062590

[31] Y. Saad, Analysis of some Krylov subspace approximations to the matrix exponential operator, SIAM Journal on Numerical Analysis 29 (1) (1992) $209 ? 28$.

URL http: //epubs.siam.org/doi/abs/10.1137/0729014

[32] Q. Ye, An adaptive block Lanczos algorithm, Numerical Algorithms 12 (1) (1996) 97-110. URL http://link.springer.com/article/10.1007/BF02141743

[33] X. Li, W. E, Boundary conditions for molecular dynamics simulations at finite temperature: Treatment of the heat bath, Phys. Rev. B 76 (2007) 104107.

[34] C. H. Bischof, G. Quintana-Ortí, Algorithm 782: codes for rank-revealing qr factorizations of dense matrices, ACM Transactions on Mathematical Software (TOMS) 24 (2) (1998) 254-257.

[35] V. Bulatov, W. Cai, Computer simulations of dislocations, Vol. 3, Oxford University Press, 2006.

[36] M. Grujicic, P. Dang, Molecular dynamics embedded atom method simulations of crack-tip transformation toughening in fer ni austenite, Materials Science and Engineering: A 199 (2) (1995) 173-182.

[37] S. Plimpton, P. Crozier, A. Thompson, LAMMPS-large-scale atomic/molecular massively parallel simulator, Sandia National Laboratories.

[38] E. G. Karpov, H. S. Park, W. K. Liu, A phonon heat bath approach for the atomistic and multiscale simulation of solids, Int. J. Numer. Meth. Engng. 70 (2007) 351-378. 